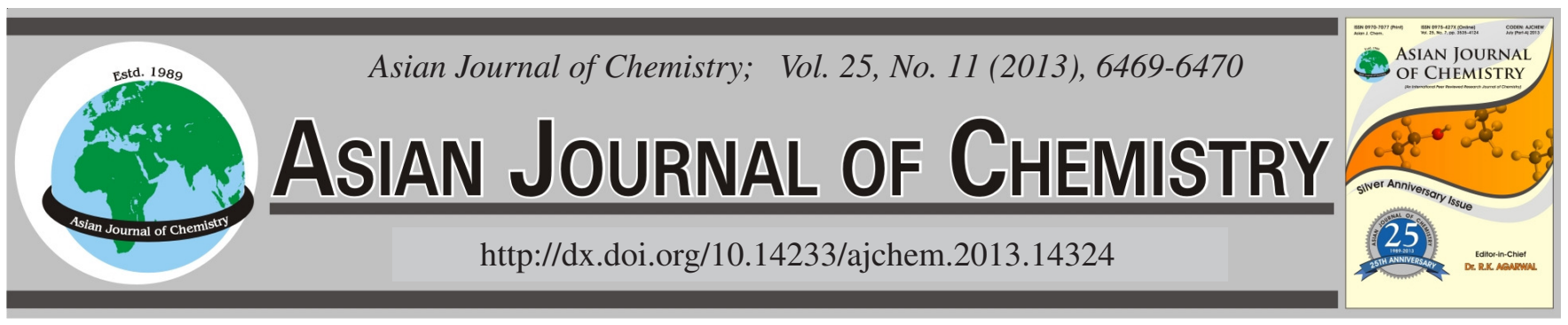

NOTE

\title{
Determination of Trace Elements and Heavy Metals in Selenium-Enriched Malt Powder by ICP-MS
}

\author{
HongXING ZhanG ${ }^{1, *}$ and YukUI RUI ${ }^{2}$
}

${ }^{1}$ Beijing Key Laboratory of Agricultural Product Safety Detection and Control, Department of Food Science, Beijing University of Agriculture, Beijing 102206, P.R. China

${ }^{2}$ College of Resources and Environmental Sciences, China Agricultural University, Beijing 100193, P.R. China

*Corresponding author: Fax: +86 10 80799170; Tel: +8610 80794124; E-mail: zhanghx511@ gmail.com

\begin{abstract}
Selenium is an essential element for human health, plays an irreplaceable role on human aging and disease prevention. Selenium-enriched malt powder is an important health care products enriched selenium, selenium - enriched malt powder should be safe as food. Today the role of other trace elements and safety of heavy metals in selenium - enriched malt powder are ignored. In this study, selenium-enriched malt powder produced by Beijing Sevkon ecological science and technology Co., Ltd was determined the trace elements and heavy metals. The results showed that the content of selenium produced by Beijing Sevkon ecological science and technology Co. Ltd. is higher than normal malt and other brands of selenium-enriched malt powder; the content of some heavy metals in enriched malt powder exceed the national standards. We should pay particular attention to their food safety. A national standard of selenium-enriched food production should be developed as soon as possible to promote the standardization and scientific production of the selenium-enriched product.
\end{abstract}

Key Words: Selenium, Selenium enriched malt powder, Heavy metals, Trace elements.

Selenium is an essential element for human health, plays an irreplaceable role on human aging and disease prevention. $72 \%$ soil of China is selenium depleted and more than 0.7 billion Chinese have selenium deficiency. Selenium enriched agricultural products which are used as the best way to supplement selenium have wide prospect and are developing rapidly. Meanwhile, scientific research institutions and government pay massive attention on the products. However because China don't have general standards about selenium enriched products and the quality of them is not uniform.

Selenium-enriched malt powder is an important health care products enriched selenium, selenium-enriched malt powder should be safe as food, can prevent many diseases, especially cancer occurrence and development ${ }^{1}$. Today, the attention of consumer and researcher is focused on the content of selenium-enriched malt powder, but the role of other trace elements and safety of heavy metals in selenium-enriched malt powder are ignored.

Inductively coupled plasma mass spectrometry (ICP-MS) is Thermo-X7 produced by Thermo Electron Corporation of USA is used in this experiment.

Preparation of samples: The samples were dried, grated; weighed $0.1 \mathrm{~g}$ sample in a small flask, soaked in $6 \mathrm{~mL}$ concen- trated nitric acid for $12 \mathrm{~h}$, then added $2 \mathrm{~mL} 30 \% \mathrm{H}_{2} \mathrm{O}_{2}$ for $4 \mathrm{~h}$; heated to boiling, transferred to a $15 \mathrm{~mL}$ centrifuge tube $(15 \mathrm{~mL}$ centrifuge tube weighing in advance) when the sample remaining $1 \mathrm{~mL}$, constant volume for testing. Three repeats were set.

Materials: In this study, selenium-enriched malt powder was produced by Beijing Sevkon ecological science and technology Co. Ltd.

Content of selenium in malt: Ping and Qin $^{2}$ prove that the content of selenium is $40-60 \mathrm{ppm}$ in selenium-enriched malt, which is 600-1000 times higher selenium content than normal wheat seed. The content of selenium in this study was 93.4 ppm, which prove that selenium-enriched malt powder produced by Beijing Sevkon ecological science and technology Co. Ltd. is better.

Other trace elements in selenium-enriched malt: The results showed that selenium-enriched malt contained more $\mathrm{Ca}, \mathrm{Fe}$ and $\mathrm{Zn}$ than normal malt (Table-1), the contents of $\mathrm{Ca}$, $\mathrm{Fe}$ and $\mathrm{Zn}$ in selenium-enriched malt were 2.65 times, 6.73 times and 18.4 times of the contents of $\mathrm{Ca}, \mathrm{Fe}$ and $\mathrm{Zn}$ in normal malt, respectively, which proved that $S$ e element can contribute to the absorption of trace elements $\mathrm{Ca}, \mathrm{Fe}$ and $\mathrm{Zn}$.

Heavy metals in selenium-enriched malt: According to the national standards GB $16740-1997^{4}$, GB $14961-94^{5}$ and 


\begin{tabular}{ccc}
\hline \multicolumn{3}{c}{ TABLE-1 } \\
\multicolumn{3}{c}{ CONTENTS OF TRACE ELEMENTS IN } \\
SELENIUM ENRICHED MALT POWDER $(\mu \mathrm{g} / \mathrm{g})$ \\
\hline Elements & Se-malt & Normal malt $^{3}$ \\
\hline $\mathrm{Ca}$ & 818.0 & 308.8 \\
$\mathrm{Fe}$ & 105.0 & 15.6 \\
$\mathrm{Zn}$ & 42.4 & 2.3 \\
\hline
\end{tabular}

GB $15201-94^{6}$, the upper limits of $\mathrm{Pb}, \mathrm{As}, \mathrm{Cr}$ and $\mathrm{Cd}$ are 0.5 , $0.3,1.0$ and $0.1 \mathrm{ma} / \mathrm{kg}$, so $\mathrm{Pb}$ and $\mathrm{Cd}$ seriously beyond the limits (Table-2), but As and Cr meet the standards.

\begin{tabular}{ccc}
\multicolumn{4}{c}{ TABLE-2 } \\
CONTENTS OF HEAVY METALS IN \\
SELENIUM ENRICHED MALT POWDER $(\mu \mathrm{g} / \mathrm{g})$ \\
\hline Elements & Se-malt & Limits of GB \\
\hline $\mathrm{Pb}$ & 4.35 & $0.50^{4}$ \\
$\mathrm{As}$ & 0.16 & $0.3^{4}$ \\
$\mathrm{Cd}$ & 0.54 & $0.1^{5}$ \\
$\mathrm{Cr}$ & 0.92 & $1.0^{6}$ \\
\hline
\end{tabular}

Gao et al. ${ }^{7}$ research suggested that PTDIs (Provisional Tolerable Daily Intake) of $\mathrm{Pb}$ and $\mathrm{Cd}$ are 225 and $63.0 \mu \mathrm{g} / \mathrm{d}$, respectively. Chinese Nutrition Society published in 2000 the appropriate intake of $\mathrm{Cr}$ is $50 \mu \mathrm{g} / \mathrm{d}$.

Chinese nutrition society recommended the safe and appropriate range of normal adult intake of selenium is 50$250 \mu \mathrm{g} / \mathrm{d}$, so the daily consumption of $535-2676 \mathrm{mg}$ the above selenium-enriched malt can meet the needs of the selenium. When consume 535-2676 mg enriched malt powder, the intake of heavy metals $\mathrm{Cr}, \mathrm{Cd}$ and $\mathrm{Pb}$ does not exceed the tolerable upper intake of the human body, so accordance with the requirements of the consumption of this malt powder is safe and harmless to the human body (Table-3).

\begin{tabular}{|c|c|c|c|}
\hline \multicolumn{4}{|c|}{$\begin{array}{c}\text { TABLE-3 } \\
\text { INTAKES OF HEAVY METALS WHEN TAKING } \\
\text { SELENIUM ENRICHED MALT POWDERS }(\mu \mathrm{g})\end{array}$} \\
\hline Consumption (mg) & $\mathrm{Cd}$ & $\mathrm{Pb}$ & $\mathrm{Cr}$ \\
\hline 535 & 0.29 & 2.33 & 0.49 \\
\hline 2676 & 1.45 & 11.65 & 2.46 \\
\hline
\end{tabular}

\section{Conclusion}

- The content of selenium produced by Beijing Sevkon ecological science and technology Co. Ltd. is higher than normal malt and other brands of selenium-enriched malt powder.

- The content of some heavy metals in enriched malt powder exceed the national standards, we should pay particular attention to their food safety.

- It is suggested that the state should develop a National Standard of selenium-enriched food production as soon as possible to promote the standardization and scientific production of the selenium-enriched product ${ }^{8}$. This research was supported by the Key National Natural Science Foundation of China (No.41130526).

\section{ACKNOWLEDGEMENTS}

This research was supported by the Key National Natural Science Foundation of China (No.41130526).

\section{REFERENCES}

1. H. Zhu, J. Mountain Agric. Biol., 18, 239 (1999).

2. P. Xiao and Q. Qin, Sci. Technol. Food Ind., 33 (1988).

3. Y.L. Miao and Y. Liu, Beer Sci. Technol., 4, 32 (1988).

4. National Standards of P.R. China, General Standard for Health (Functional) Foods, GB 16740 (1997).

5. National Standards of P.R. China, Tolerance Limit of Chromium in Foods, GB 14961-94.

6. National Standards of P.R. China, Tolerance Limit of Cadmium in Foods, GB 15201-94.

7. J.Q. Gao, X.W. Li and J.L. Zhao, J. Hygiene Res., 35, 750 (2006).

8. X.J. Zheng, China Standardization, 5, 19 (2003). 